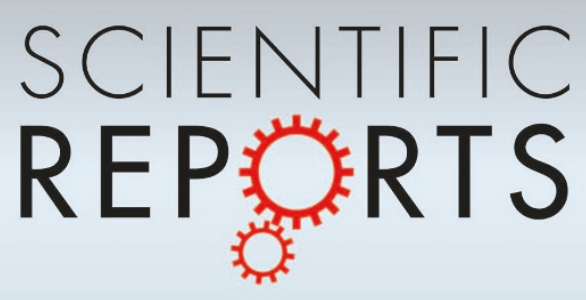

OPEN

SUBJECT AREAS:

SPINTRONICS

ELECTRONIC PROPERTIES AND

DEVICES

Received

23 April 2014

Accepted

2 September 2014

Published

24 September 2014

Correspondence and requests for materials should be addressed to J.-F.L. (liu.j¡@sustc.edu. $\mathrm{cn})$

\title{
Equilibrium spin current in graphene with Rashba spin-orbit coupling
}

\author{
Huan Zhang' ${ }^{1}$ Zhongshui $\mathrm{Ma}^{2,3}$ \& Jun-Feng Liu'
}

'Department of Physics, South University of Science and Technology of China, Shenzhen 518055, P.R. China, ${ }^{2}$ School of Physics,
Peking University, Beijing 100871, P.R. China, ${ }^{3}$ Collaborative Innovation Center of Quantum Matter, Beijing, 100871, P.R. China.

The existence of a background spin current under thermodynamic equilibrium is an interesting phenomenon in the two-dimensional electron gas with Rashba spin-orbit coupling (RSOC). Here we study the equilibrium spin current (ESC) in graphene with RSOC. For an infinite graphene with uniform RSOC, we found that the ESC is proportional to $\lambda^{2}$ with $\lambda$ the Rashba strength and mainly comes from the energy window $[-\lambda, \lambda]$ near Dirac points. In the regime of energy far away from Dirac points, the $\lambda^{3}$ dependence as that in a normal two-dimensional electron gas is recovered. In a system with a normal graphene strip inserted between two Rashba graphene sheets, we found that the ESC can penetrate through the normal graphene layer (perpendicular to the interface). This unique effect can be understood by considering the spin-filtered scattering from the normal region to the RSOC region. The finding of the ESC through the normal region without RSOC advances the understanding of ESC and provides a new way to generate a pure spin current in graphene. For an experimentally accessible strength of Rashba spin-orbit coupling, the ESC remains over room temperature.

raphene is a promising candidate for spintronics and related applications due to weak intrinsic spin-orbit
coupling ${ }^{1-3}$ and low hyperfine interactions ${ }^{4}$. The measured values of the spin-coherence length in gra-
phene reach $1.5-2 \mu \mathrm{m}^{5}$, which promotes graphene as a spin current conductor of high performance.
Furthermore, externally induced large Rashba spin-orbit coupling (RSOC) was reported recently ${ }^{6}$, which is
opening the possibility of using graphene in active elements of spintronics devices. The reported giant Rashba
splitting reaches $100 \mathrm{meV}$ in the Au-intercalated graphene-Ni system. Motivated by the experimental develop-
ments, a lot of theoretical works ${ }^{7-16}$ were devoted to investigating the effect of RSOC on the transport properties of
graphene.

Generating spin currents in graphen $\mathrm{e}^{17-19}$ is one of the central goals of graphene-based spintronics. In the normal two-dimensional electron gas (2DEG) with RSOC, the existence of a background spin current under thermodynamic equilibrium has caused much controversy about the spin-current concept ${ }^{20-24}$. The spin current in equilibrium was regarded as a byproduct of the application of standard spin-current definition in the Rashba medium where the spin is not conserved ${ }^{20}$. A wide discussion was triggered on the spin-current definition ${ }^{25-29}$. And many proposals were designed to observe the equilibrium spin current (ESC) ${ }^{26,30-33}$. The ESC found in this work may be detected by the induced mechanical torques on a substrate at edges of the Rashba medium ${ }^{30}$, spincurrent-induced electric field ${ }^{31}$, a polarized light beam ${ }^{32}$, and the induced second-order nonlinear optical effect ${ }^{33}$. The reported giant Rashba splitting in graphene made this field revitalized. In graphene, RSOC is dominated by the coupling between real spin and pseudospin instead of the orbital momentum near Dirac cones ${ }^{8,34}$. Consequently, a graphene-bilayer-like spin-splitting is caused in graphene and is very different from that in a normal 2DEG. Some unusual behaviors of ECS could, therefore, be expected in graphene with RSOC. To the best of our knowledge, the relevant study is still lacking.

In this work, the ESC in graphene with RSOC is studied by employing both analytical and numerical calculations. It is shown that the dependence of ESC on Rashba strength $\lambda$ is of the second order in graphene, which is significantly different from a $\lambda^{3}$ dependence in the normal 2DEG ${ }^{20}$. The ESC comes mainly from the energy region $[-\lambda, \lambda]$ near the Dirac points where only one spin subband exists. Consequently, the ESC depends dramatically on the chemical potential $\mu$ and reaches the maximum at the charge-neutral Dirac point. We found that the ESC can penetrate through a Rashba-normal-Rashba (RNR) graphene junction in which a normal graphene strip is sandwiched by two Rashba graphenes (see Fig. 1 (a) and (b)). The phenomenon of ESC through a region without RSOC can be understood as the effect of spin-filtered scattering from the normal region to the Rashba region and is related to the formation of discrete bound states which transfer spin in the normal region. This ESC through a region without RSOC where the definition of spin current is unambiguous indicates that the existence of ESC is 


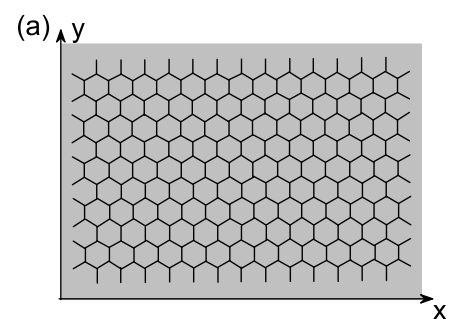

(c) $\uparrow \mathrm{E}$

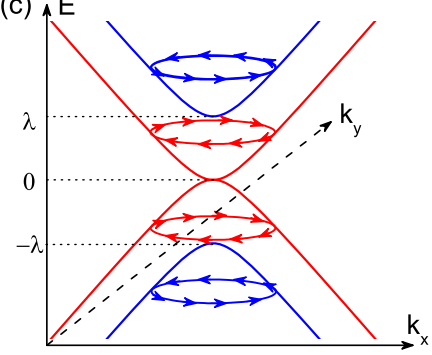

(b)

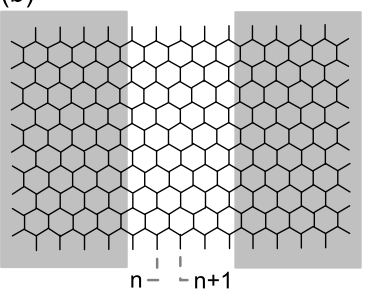

(d)

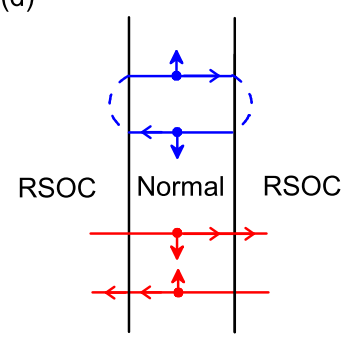

Figure 1 Schematic illustrations of an infinite graphene with RSOC and a graphene RNR junction, band structure of graphene with RSOC, schematic illustrations of bound and propagating states in a RNR junction. (a) and (b) Schematic illustrations of infinite graphene with RSOC (a) and RNR graphene junction (b). RSOC is present in shade areas. (c) Four subbands with different indexes of spin and pseudospin, the arrows mark the spin directions. (d) Schematic illustrations of two kinds of states in the normal graphene region of the RNR junction in the energy range $[0, \lambda]$ : right-going spin-up electron and left-going spin-down one form discrete bound states, while right-going spin-down electron and leftgoing spin-up one are well transmitted into RSOC regions.

not concerned with the conservativeness of spin. It advances the understanding of ESC and provides a new way in the generation of a pure spin current in graphene systems.

\section{Results}

States near Dirac points. The energy region $[-\lambda, \lambda]$, where only one spin subband exists, plays an important role in producing the ESC. The band spectrum, especially the spin structure near Dirac points in graphene with RSOC is crucial to the produced ESC. Near Dirac points, the Hamiltonian for graphene with RSOC is given by ${ }^{8}$

$$
H=\gamma\left(\tau k_{x} \sigma_{x}+k_{y} \sigma_{y}\right)+\frac{\lambda}{2}\left(\tau \sigma_{x} s_{y}-\sigma_{y} s_{x}\right)
$$

where $\gamma=\hbar v$ and $v \approx 10^{8} \mathrm{~cm} / \mathrm{s}$. Here $\sigma$ and $\mathrm{s}$ are Pauli matrices representing the pseudospin and the real spin, respectively. $\tau= \pm 1$ describes states at two inequivalent Dirac cones.

The eigenstates of the four subbands are found as

$$
|\sigma s\rangle_{R}=\frac{1}{\sqrt{2\left(1+\eta^{2 \tau}\right)}}\left(\begin{array}{c}
1 \\
s i e^{i \theta} \eta^{\tau} \\
\tau \sigma e^{i \tau \theta} \eta^{\tau} \\
\tau \sigma s i e^{i(1+\tau) \theta}
\end{array}\right)
$$

where $\sigma= \pm 1$ describes electrons or holes and $s= \pm 1$ describes two spin subbands, $\theta$ is the orientation angle of $\mathbf{k}, \eta=\frac{|E|}{\gamma k}$, and the subscript $R$ denotes the presence of RSOC. They correspond to the eigenvalues

$$
E=\sigma\left(\sqrt{\gamma^{2} k^{2}+\frac{\lambda^{2}}{4}}+s \frac{\lambda}{2}\right)
$$

where $k=\sqrt{k_{x}^{2}+k_{y}^{2}}$. In the limit $\gamma k \ll \frac{\lambda}{2}$, Eq. (3) reduces to $E=\sigma\left[\frac{\gamma^{2} k^{2}}{\lambda}+(1+s) \frac{\lambda}{2}\right]$, which is similar to the parabolic spectrum for a bilayer graphene. The term $\pm \frac{\lambda}{2}$ manifests the effect of coupling between real spin and pseudospin in a Zeeman splitting. Conversely, in the limit $\gamma k \gg \frac{\lambda}{2}, E=\sigma\left(\gamma k+s \frac{\lambda}{2}\right)$. The linear spectrum is recovered and the spin-splitting has a form as that in the normal 2DEG.

Employing the eigenstates, the spin averages for the four subbands are found as

$$
\left\langle s_{x}\right\rangle_{\sigma s}=-s \frac{2 \eta \sin \theta}{1+\eta^{2}},\left\langle s_{y}\right\rangle_{\sigma s}=s \frac{2 \eta \cos \theta}{1+\eta^{2}},\left\langle s_{z}\right\rangle_{\sigma s}=0 .
$$

Thus, $\mathbf{k} \cdot \mathbf{s}=0$. It means that the spin is perpendicular to the wavevectors for every subband as illustrated in Fig. 1 (c). Eq. (4) indicates that the band-average spin vector is independent of both $\sigma$ and $\tau$.

The spin current of a given state $\left|\psi_{\sigma s, \mathbf{k}}\right\rangle$ is defined by

$$
\mathcal{J}_{i j}=\frac{\hbar}{4}\left\langle\psi_{\sigma s, \mathbf{k}}\left|s_{i} v_{j}+v_{j} s_{i}\right| \psi_{\sigma s, \mathbf{k}}\right\rangle
$$

where the velocity operator $v_{j}=\hbar^{-1} \partial H / \partial k_{j}, i=x, y, z$ represent the spin components, and $j=x, y$ represent the transfer directions of spin carriers. There exist some symmetric properties among the components of the total spin current as follows

$$
J_{y x}=-J_{x y}, J_{x x}=J_{y y}=0, J_{z x}=J_{z y}=0 .
$$

So we need to consider $J_{y x}$ only. Because $v_{x}=\hbar^{-1} \gamma \tau \sigma_{x}$ and $\sigma_{x}$ commutes with $s_{y}$, the spin current operator $\hat{\mathcal{J}}_{y x}$ takes form as $\hat{\mathcal{J}}_{y x}=\gamma \tau s_{y} \sigma_{x} / 2$. For a given $\mathbf{k}$, the contribution from subband $E_{\sigma s}$ is found to be

$$
\mathcal{J}_{y x}=\frac{\sigma s \gamma}{2} \frac{\eta^{2}+\cos 2 \theta}{1+\eta^{2}} .
$$

This implies that $K$ and $K^{\prime}$ valleys have the same contribution to the total spin current $J_{y x}$. The total contribution of each subband $|\sigma s\rangle_{R}$ to $J_{y x}$ in a given energy region can be obtained by integrating Eq. (7) over an annulus in the $\mathbf{k}$ space

$$
\begin{aligned}
& \frac{1}{(2 \pi)^{2}} \int_{k_{1}}^{k_{2}} \int_{0}^{2 \pi} \mathcal{J}_{y x} k d \theta d k \\
= & \frac{\sigma s}{8 \pi \gamma} \int_{E\left(k_{1}\right)}^{E\left(k_{2}\right)} E d E=\frac{\sigma s}{16 \pi \gamma}\left[E^{2}\left(k_{2}\right)-E^{2}\left(k_{1}\right)\right],
\end{aligned}
$$

where $\gamma^{2} k^{2}=E^{2}-\sigma s E \lambda$ is used. Here $k_{1}$ and $k_{2}$ are two arbitrary positive real numbers and $k_{1}<k_{2}$, so that $E^{2}\left(k_{2}\right)-E^{2}\left(k_{1}\right)>0$ is guaranteed.

Only the subband $E_{\sigma, s=-1}$ occurs in the energy window $[-\lambda, \lambda]$. The contribution from $|\sigma, s=-1\rangle_{R}$ to $J_{y x}$ is found as $-\sigma \lambda^{2} / 16 \pi \gamma$ in the energy window $[-\lambda, \lambda]$. Obviously, its dependence on the chemical potential $\mu$ for $T=0$ manifests a down opening parabola and reaches the maximum at $\mu=0$. It shows a $\lambda^{2}$-dependence in the spin current. Out of the energy window $[-\lambda, \lambda]$, both subbands with $s=$ \pm 1 exist. Their contributions to $J_{y x}$ are cancelled. In the energy region far away from Dirac points, the contribution to the ESC should be proportional to $\lambda^{3}$, similar to that in a normal 2DEG. Therefore, the contribution from the energy window $[-\lambda, \lambda]$ is dominant in the ESC. This will also be demonstrated in the following numerical calculations. It is noticeable that the Rashba strength is supposed to be positive $(\lambda>0)$ throughout this paper. In the case of $\lambda$ $<0$, all the results of spin current will acquire a sign change because 
the subband $E_{\sigma, s=1}$ occurs in the energy window $[-|\lambda|,|\lambda|]$ instead according to Eq. (3).

There exists also a spin-filtering effect occurring at the normal/ RSOC interface which leads to the penetration of the ESC into the normal graphene region in a RNR junction. The spin-filtering effect also stems from the fact that only one spin subband is open in the energy window $[-\lambda, \lambda]$. We consider an electron incident from a normal graphene region $(x<0)$ towards to the RSOC region $(x>0)$ along the $x$-axis. The state is described by $\langle\sigma s\rangle_{N}=\frac{1}{2}\left(1, s i, \tau \sigma e^{i \tau \theta}, \tau \sigma s i e^{i \tau \theta}\right)^{T}$, where $T$ stands for a transpose of the matrix. Its spin is either parallel or antiparallel to the $y$-axis. The modulus of the overlap between this incident state and the existing state $\left|\sigma^{\prime}-1\right\rangle_{R}$ in the energy window $[-\lambda, \lambda]$ reads

$$
\begin{aligned}
& \left|{ }_{R}\left\langle\sigma^{\prime}-1 \mid \sigma s\right\rangle_{N}\right| \\
= & \frac{1}{2 \sqrt{2\left(1+\eta^{2}\right)}}\left|1-\sigma \sigma^{\prime} s e^{i\left(\theta-2 \theta^{\prime}\right)}+\eta e^{-i \theta^{\prime}}\left(\sigma \sigma^{\prime} e^{i \theta}-s\right)\right|
\end{aligned}
$$

which is independent of $\tau$. For simplicity, we consider the case of normal incidence, i.e., $k_{y}=0$, and assume $\sigma=\sigma^{\prime}$. This implies $\theta=\theta^{\prime}$ $=0, \pi$. Consequently, the modulus then becomes

$$
\left|{ }_{R}\left\langle\sigma^{\prime}-1 \mid \sigma s\right\rangle_{N}\right|=\frac{1}{2 \sqrt{2\left(1+\eta^{2}\right)}}(1+\eta)(1-s \sigma v)
$$

where $v= \pm 1$ is the sign of the velocity. For electrons, $\sigma=1$, Eq. (10) implies that only right-going spin-down (with respect to the $y$-axis) and left-going spin-up electrons can enter the RSOC region. The right-going spin-up and left-going spin-down electrons are forbidden. For holes, $\sigma=-1$, the situation is reversed.

Equilibrium spin current. The ESC is numerically calculated based on the tight-binding model. For an infinite graphene with uniform RSOC, Fig. 2 (a) shows the dependence of energy-resolved ESC $j_{y x}$ on the energy $E$ for various $\lambda . j_{y x}$ is the summation of single-state contribution $\mathcal{J}_{y x}$ over the momentum space for a fixed energy. Numerically, $j_{y x}$ is evaluated by Eq. (13) using Green's function technique described in the Methods section. The unit is $j_{0}=1 /$ $24 a \pi^{2}$. For a given energy, $j_{y x}$ includes the contributions from all the modes. The curves clearly indicate three region for the energy dependence of ESC. Firstly, the contribution from the energy range $[-\lambda, \lambda]$ is dominant. $j_{y x}$ behaves in a linear dependence on $E$ near Dirac point. Away from the range $[-\lambda, \lambda], j_{y x}$ almost vanishes until the neighborhood of $E= \pm t$. These features are consistent with the previous conclusion from the discussion on Eq. (8). Besides the energy window $[-\lambda, \lambda]$, there are some contributions to ESC for the energies around $E= \pm t$ ( $M$ points) and near $E= \pm 3 t$ ( $\Gamma$ points). We plot total ESC $J_{y x}$ against the chemical potential $\mu$ in Fig. 2 (b) at room temperature where the unit is $J_{0}=\mathrm{meV} / 6 \pi \mathrm{a}$. In the energy window $[-\lambda, \lambda], J_{y x}$ exhibits a down opening parabola and reaches its maximum value at $\mu=0$ as we have discussed previously. The maximum at $\mu=0$ is proportional to $\lambda^{2}$. The value of $J_{y x}$ just outside the energy window $[-\lambda, \lambda]$ is proportional to $\lambda^{3}$. Such a $\lambda^{3}$ dependence reflects the contribution from the neighborhood of $M$ and $\Gamma$ points. The temperature dependence of $J_{y x}$ has also been shown in the inset of Fig. 2 (b) for $\lambda=90 \mathrm{meV}$.

ESC through RNR junctions. More important, the ESC can penetrate through the normal graphene layer without RSOC in a RNR graphene junction. Fig. 3 shows $j_{y x}$ for various number of columns in the normal region. The sharp dips or peaks in $j_{y x}$ correspond to the bound states emerging at $k_{y}=0$ because the component of $k_{y}=0$ is dominant. As will be discussed later, the bound states consist of right-going spin-up and left-going spin-down electrons when $E>0$. If the width of normal graphene strip is very small, there exists only one bound state on the edges of the energy
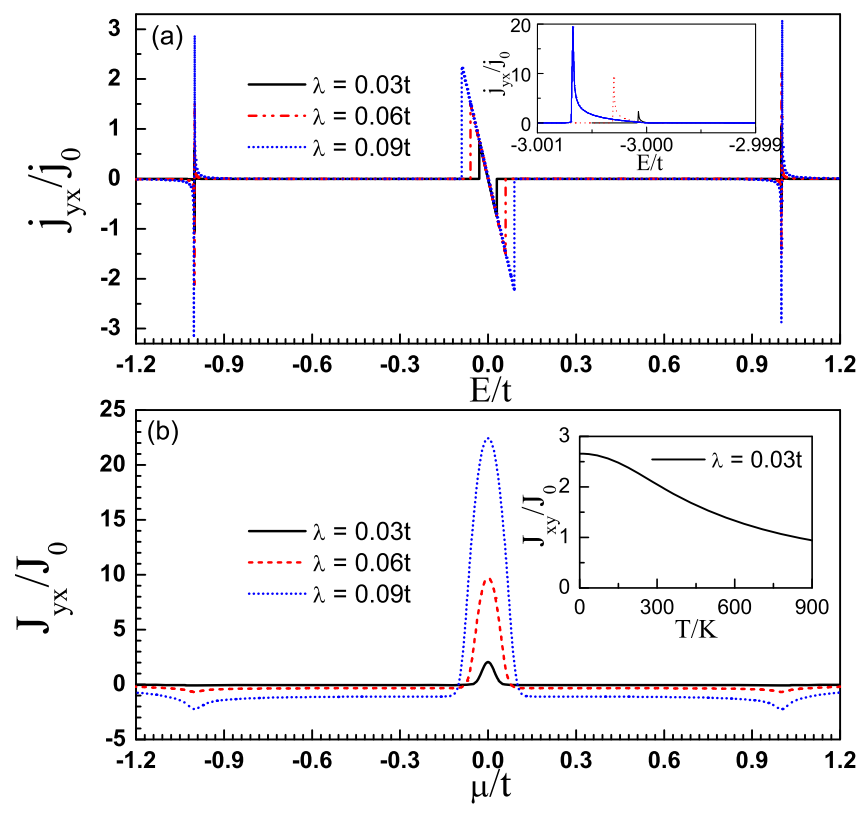

Figure $2 \mid$ Energy-resolved ESC and the total ESC for an infinite graphene with uniform RSOC. (a) $j_{y x}$ versus $E$ for various $\lambda$, the area around $E=-3 t$ is shown in the insert. (b) $J_{y x}$ versus $\mu$ at $T=300 \mathrm{~K}$, the insert shows the temperature dependence of $J_{y x}$ for $\lambda=90 \mathrm{meV}$.

window $[-\lambda, \lambda]$. Thus, the contribution to the ESC from the bound states is weak. With increasing width of the normal region, the bound state moves to the central $E=0$ and makes more contribution to $J_{y x}$. Consequently, $J_{y x}$ is partly suppressed. When the width is up to $N=$ 100 , the second bound state is involved in the energy window $[-\lambda, \lambda]$ and with the result that $J_{y x}$ is reduced sensitively.

Fig. 4 (a) plots $J_{y x}$ in the normal region versus $\mu$ for different widths of the normal region $N$ at $T=300 \mathrm{~K}$. We found that the contribution from the energy window $[-\lambda, \lambda]$ is again dominant in $J_{y x}$. With increasing $N, J_{y x}$ decreases exponentially as shown in Fig. 4 (b). For larger $N, J_{y x}$ in the normal region can be reversed in sign by tuning $\mu$. When $N$ is up to $50, J_{y x}$ at $\mu=0$ decreases to $1 / 1000$ of the value for $N$ $=0$. The spatial distribution of $J_{y x}$ in the whole RNR junction is shown in Fig. 4 (c). The ESC shrinks rapidly before entering into the normal region. It keeps constant in the normal region due to the absence of spin source. The temperature dependence of $J_{y x}$ in the normal region is shown in Fig. 4 (d) for $\mu=0$ and $\lambda=90 \mathrm{meV}$. Because the contribution from $E>0$ is opposite to that from $E<0$ (shown in Fig. 3), $J_{y x}$ decreases with increasing temperature due to the Fermi-Dirac distribution. It is shown that $J_{y x}$ decreases more quickly for larger $N$. This is because that more bound states result in heavier oscillation in $j_{y x}$ as the function of energy. For smaller $N$, the ESC in the normal region can remain over room temperature.

We have also investigated the effect of imperfect interfaces by considering a finite interface barrier at two interfaces between the normal graphene and Rashba graphene. The spin current $J_{y x}$ with various interface barrier strength $V_{B}$ is shown in Fig. 5 . It shows that $J_{y x}$ can survive even with a strong interface barrier. At chemical potential $\mu=0, J_{y x}$ experiences a sign change when the interface barrier increases because the propagation state is suppressed and the position of bound state moves.

\section{Discussion}

All the main results of this paper can be understood by the analysis of the states near Dirac points. As we have seen, the numerical results of ESC in an uniform Rashba graphene agree very well with our analytical result (Eq. (8)) which is based on the states near Dirac points. 


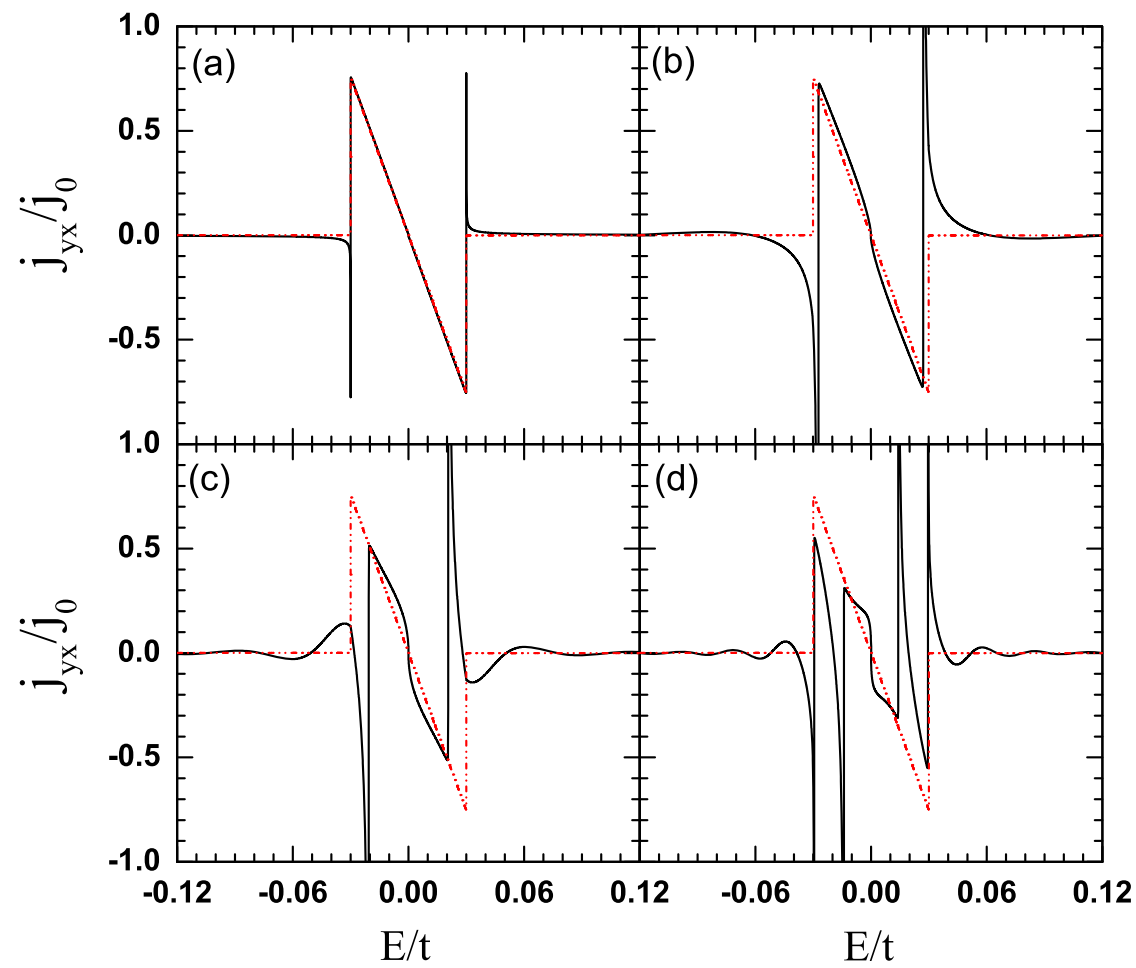

Figure 3 Energy-resolved ESC $\boldsymbol{j}_{y x}$ in the normal region of RNR junction for various width of the normal region. The width of the normal region $N$ is: (a) 1 , (b) 20, (c) 50, (d) 100. $\lambda=90 \mathrm{meV}$. For a reference, the result of the uniform Rashba grapene $(N=0)$ is shown in dotted line.

Furthermore, the novel ESC through the normal region can be understood by considering the spin-filtered scattering from the normal region to the RSOC region and is related to the formation of discrete bound states which transfer spin in the normal region. For simplicity, we focus on behaviors of electrons, which corresponds to the energy range $[0, \lambda]$. For holes, the situation is reversed. From Eq. (10) we have learned that right-going spin-down (with respect to the $y$-axis) and left-going spin-up electrons can be well transmitted into
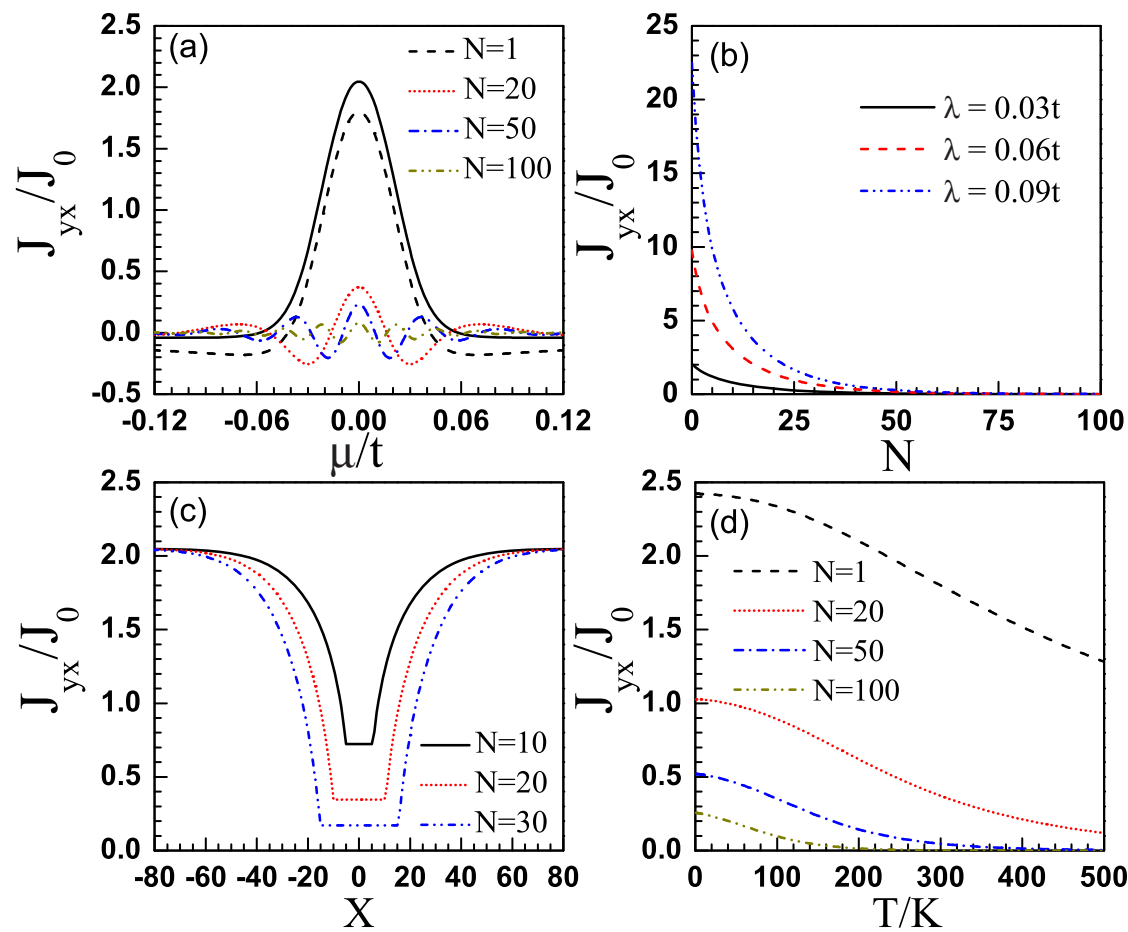

Figure $4 \mid$ The total ESC $J_{y x}$ in the normal region of a RNR junction. (a) $J_{y x}$ versus $\mu$ for various width of the normal region $N$ with $T=300 \mathrm{~K}$ and $\lambda=90 \mathrm{meV}$. The results of $\mathrm{N}=50$ and 100 have been multiplied by a factor, 5 and 50 respectively. The solid line is for the case of $N=0$. (b) $J_{y x}$ versus $N$ for various $\lambda$ with $T=300 \mathrm{~K}, \mu=0$. (c) $J_{y x}$ as the function of the position in RNR junctions with various $N, \lambda=90 \mathrm{meV}, \mu=0$. (d) $J_{y x}$ versus $T$ for various $N$, $\lambda=90 \mathrm{meV}, \mu=0$. 


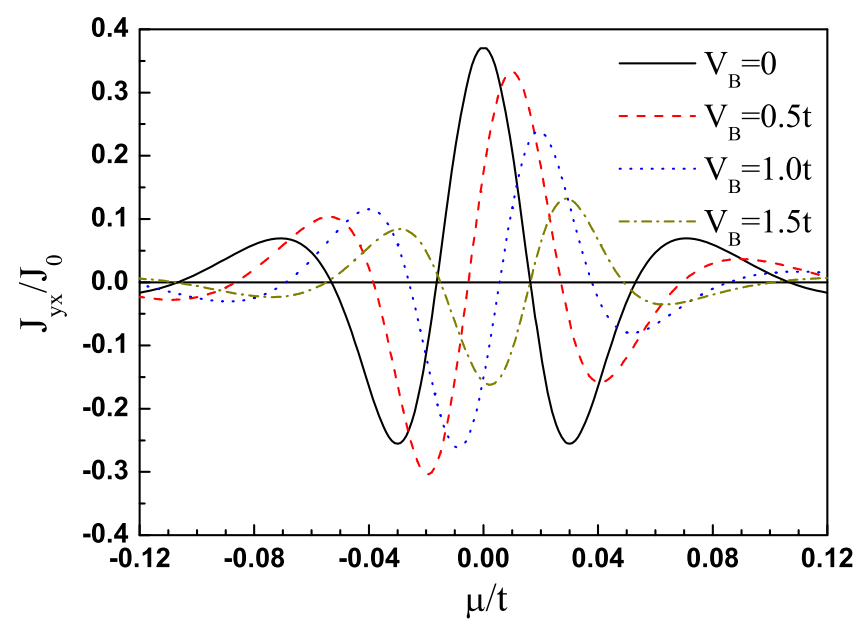

Figure $5 \mid J_{y x}$ versus $\mu$ for the RNR junction with several choices of interface barrier strength $V_{B}$. Here $T=300 \mathrm{~K}, N=20$ and $\lambda=90 \mathrm{meV}$.

the RSOC region while right-going spin-up and left-going spin-down electrons would be reflected completely. The analytical calculations show that right-going spin-up electrons are completely reflected to left-going spin-down electrons and vice versa. Therefore right-going spin-up and left-going spin-down electrons form bound states with discrete energies in the strip of normal graphene (illustrated in Fig. 1 (d)). For $k_{y}=0$, the energies of these bound states satisfy the condition

$$
\arccos \left(1-\frac{2|E|}{\lambda}\right)+\frac{1}{\gamma}|E| L=n \pi,
$$

where $L$ is the width of the normal region and $n$ is an arbitrary integer. This analytical expression of bound states gives the dependence of bound state energies on the length of the normal layer and agrees very well with the numerical calculation.

The continuous states, right-going spin-down and left-going spinup electrons, make negative contribution to $J_{y x}$ while the discrete bound states make positive contribution to $J_{y x}$. When the length of the normal graphene increases, more bound states occur in the energy windows $[-\lambda, \lambda]$ and make more compensative contribution. Hence the ESC through the normal region is suppressed more heavily. Outside the energy window, there only exist the continuous states for electrons with arbitrary spin and momentum. Far from the energy window, the transmission from the normal region to the Rashba region has no difference between spin-up and spin-down electrons, i.e., the spin-filtering effect vanishes. Then the ESC through the normal region also vanishes. This penetration effect of the ESC into the normal graphene in a RNR junction is also attributed to the properties of the states near Dirac points.

In summary, the properties of ESC in graphene with RSOC is studied. It is shown that the ESC is proportional to $\lambda^{2}$ with $\lambda$ the Rashba strength, which differs from a $\lambda^{3}$-dependence in the normal 2DEG. Our analytical and numerical calculations show unambiguously that the main contribution to the ESC comes from the energy window $[-\lambda, \lambda]$. For the region far away from Dirac points, the $\lambda^{3}-$ dependence is recovered because of the transition from Dirac particles to nonrelativistic particles. What is more interesting is that the ESC can penetrate through a region without RSOC in the RNR graphene junction. This unique effect is related to the formation of discrete bound states which transfer spin in the normal region. The finding of the ESC though the normal region without RSOC in this work advances the understanding of ESC and provides a new way to generate a pure spin current in graphene.

\section{Methods}

To take into account the states far away from Dirac points in the calculations of the ESC, we employ the tight-binding Hamiltonian on honeycomb lattice with RSOC ${ }^{34-36}$

$$
H=-t \sum_{\langle i j\rangle} c_{i}^{\dagger} c_{j}+i \frac{\lambda}{3 a} \sum_{\langle i j\rangle} c_{i}^{\dagger}\left[\mathbf{s} \times \mathbf{d}_{i j}\right]_{z} c_{j},
$$

where $\langle i, j\rangle$ denotes the summation over the nearest neighbor sites, $t(=3 \mathrm{eV}$ ) is the hopping integral for nearest neighbors, and $c_{i}^{\dagger}\left(c_{i}\right)$ represents the creation (annihilation) operator of electrons on the site $i$ neglecting the spin degree of freedom. In the Rashba term, $\mathbf{d}_{i j}$ is the displacement vector between the lattice sites $i$ and $j$, and $a$ is the $\mathrm{c}-\mathrm{c}$ bond length.

We use a unified formalism, the lattice Green's function, to calculate the ESC in graphene with uniform RSOC and the RNR junction (see Fig. 1 (a) and (b)). Obviously, $k_{y}$ is a conservative quantity. For a given $E$, the spin current from column $n$ to column $n+1$ (the labelling is shown in Fig. 1 (b)) is ${ }^{37}$

$$
j_{y x}=\frac{\hbar}{4 \pi} \int \operatorname{Tr}\left[\hat{t}_{n+1, n}^{\dagger} s_{y} G_{n+1, n}^{<}-s_{y} \hat{t}_{n+1, n} G_{n, n+1}^{<}\right] d k_{y},
$$

where $\hat{t}_{n+1, n}$ is the hopping matrix from column $n$ to column $n+1$. In equilibrium, the lesser-than Green's function is given as $G^{<}=G^{a}-G^{r}$. The advanced (retarded) Green's function $G^{a(r)}$ can be calculated by the recursive method. At finite temperature, the total spin current $J_{y x}$ is obtained by

$$
J_{y x}=\int f(E) j_{y x}(E) d E
$$

with $f(E)$ the Fermi-Dirac distribution function. Except for the effect of Fermi-Dirac distribution, other types of scattering processes such as electron-phonon interaction may also affect the spin current at a high temperature. Here we focus on the temperature range below the room temperature and suppose that the the Rashba-splitting is much larger than the temperature broadening. Thus we only consider the effect of Fermi distribution and ignore other high temperature effects.

1. Huertas-Hernando, D., Guinea, F. \& Brataas, A. Spin-orbit coupling in curved graphene, fullerenes, nanotubes, and nanotube caps. Phys. Rev. B 74, 155426 (2006).

2. Min, H. et al. Intrinsic and Rashba spin-orbit interactions in graphene sheets. Phys. Rev. B 74, 165310 (2006).

3. Yao, Y., Ye, F., Qi, X. L., Zhang, S. C. \& Fang, Z. Spin-orbit gap of graphene: Firstprinciples calculations. Phys. Rev. B 75, 041401(R) (2007).

4. Trauzettel, B., Bulaev, D. V., Loss, D. \& Burkard, G. Spin qubits in graphene quantum dots. Nat. Phys. 3, 192 (2007).

5. Tombros, N., Jozsa, C., Popinciuc, M., Jonkman, H. T. \& van Wees, B. J. Electronic spin transport and spin precession in single graphene layers at room temperature. Nature 448, 571 (2007).

6. Marchenko, D. et al. Giant Rashba splitting in graphene due to hybridization with gold. Nat. Commun. 3, 1232 (2012).

7. Zarea, M. \& Sandler, N. Rashba spin-orbit interaction in graphene and zigzag nanoribbons. Phys. Rev. B 79, 165442 (2009).

8. Rashba, E. I. Graphene with structure-induced spin-orbit coupling: Spinpolarized states, spin zero modes, and quantum Hall effect. Phys. Rev. B 79, 161409(R) (2009).

9. Bercioux, D. \& De Martino, A. Spin-resolved scattering through spin-orbit nanostructures in graphene. Phys. Rev. B 81, 165410 (2010).

10. Tse, W.-K., Qiao, Z., Yao, Y., MacDonald, A. H. \& Niu, Q. Quantum anomalous Hall effect in single-layer and bilayer graphene. Phys. Rev. B 83, 155447 (2011).

11. Liu, J., Wang, B., Ma, Z. \& Zhang, C. Two-color terahertz response in bilayer graphene nanoribbons with spin-orbit coupling. Appl. Phys. Lett. 98, 061107 (2011).

12. Liu, J.-F., Chan, K. S. \& Wang, J. A bipolar spin-filtering effect in graphene zigzag nanoribbons with spin-orbit coupling. Nanotechnology 23, 095201 (2012).

13. Liu, J.-F. \& Chan, K. S. Y-Shaped Spin Filter in Graphene with Rashba Spin-Orbit Coupling. J. Phys. Soc. Jpn. 82, 074711 (2013).

14. Liu, M.-H., Bundesmann, J. \& Richter, K. Spin-dependent Klein tunneling in graphene: Role of Rashba spin-orbit coupling. Phys. Rev. B 85, 085406 (2012).

15. Zhang, Q., Chan, K. S., Lin, Z. \& Liu, J.-F. Spin transport in graphene spin-orbit barrier structure. Phys. Lett. A 377, 632 (2013).

16. Shakouri, K., Masir, M. R., Jellal, A., Choubabi, E. B. \& Peeters, F. M. Effect of spinorbit couplings in graphene with and without potential modulation. Phys. Rev. B 88, 115408 (2013).

17. Zhang, Q., Chan, K. S. \& Lin, Z. Spin current generation by adiabatic pumping in monolayer graphene. Appl. Phys. Lett. 98, 032106 (2011).

18. Liu, J.-F. \& Chan, K. S. Spin-polarized quantum pumping in bilayer graphene. Nanotechnology 22, 395201 (2011).

19. Zhang, Q., Liu, J.-F., Lin, Z. \& Chan, K. S. Generation of large spin currents in graphene using adiabatic quantum pumping. J. Appl. Phys. 112, 073701 (2012)

20. Rashba, E. I. Spin currents in thermodynamic equilibrium: The challenge of discerning transport currents. Phys. Rev. B 68, 241315(R) (2003). 
21. Wang, J. \& Chan, K. S. Equilibrium spin current through tunneling junctions. Phys. Rev. B 74, 035342 (2006).

22. Sonin, E. B. Equilibrium spin currents in the Rashba medium. Phys. Rev. B 76, 033306 (2007)

23. Sonin, E. B. Spin currents and spin superfluidity. Adv. Phys. 59, 181 (2010).

24. Sablikov, V. A., Sukhanov, A. A. \& Tkach, Y. Y. Equilibrium edge spin currents in two-dimensional electron systems with spin-orbit interaction. Phys. Rev. B 78, 153302 (2008)

25. Shi, J., Zhang, P., Xiao, D. \& Niu, Q. Proper definition of spin current in spin-orbit coupled systems. Phys. Rev. Lett. 96, 076604 (2006).

26. Sun, Q.-F., Xie, X. C. \& Wang, J. Persistent spin current in a mesoscopic hybrid ring with spin-orbit coupling. Phys. Rev. Lett. 98, 196801 (2007).

27. Sun, Q.-F., Xie, X. C. \& Wang, J. Persistent spin current in nanodevices and definition of the spin current. Phys. Rev. B 77, 035327 (2008).

28. Drouhin, H.-J., Fishman, G. \& Wegrowe, J.-E. Spin currents in semiconductors: Redefinition and counterexample. Phys. Rev. B 83, 113307 (2011).

29. An, Z., Liu, F. Q., Lin, Y. \& Liu, C. The universal definition of spin current. Sci. Rep. 2, 388 (2012).

30. Sonin, E. B. Proposal for measuring mechanically equilibrium spin currents in the Rashba medium. Phys. Rev. Lett. 99, 266602 (2007).

31. Sun, Q.-F., Guo, H. \& Wang, J. Spin-current-induced electric field. Phys. Rev. B 69 054409 (2004).

32. Wang, J., Zhu, B.-F. \& Liu, R.-B. Proposal for direct measurement of a pure spin current by a polarized light beam. Phys. Rev. Lett. 100, 086603 (2008).

33. Werake, L. K. \& Zhao, H. Observation of second-harmonic generation induced by pure spin currents. Nat. Phys. 6, 875 (2010).

34. Kane, C. L. \& Mele, E. J. Quantum spin Hall effect in graphene. Phys. Rev. Lett. 95, 226801 (2005).

35. Kane, C. L. \& Mele, E. J. $Z_{2}$ topological order and the quantum spin hall effect. Phys. Rev. Lett. 95, 146802 (2005).

36. Haldane, F. D. M. Model for a quantum Hall effect without Landau levels: Condensed-matter realization of the "parity anomaly" Phys. Rev. Lett. 61, 2015 (1988).
37. Wang, J. \& Chan, K. S. Spin density induced by equilibrium spin current in a magnetic field. J. Phys.: Condens. Matter 21, 026001 (2009).

\section{Acknowledgments}

The work described in this paper is supported by the National Natural Science Foundation of China (NSFC, Grant No. 11204187, 11204185, 11274013) and NBRP of China (2012CB921300).

\section{Author contributions}

J.F.L. conceived the study and made the analytical calculation. H.Z. performed the numerical calculations. Z.S.M. gave scientific advice. J.F.L. wrote the main manuscript text and Z.S.M. made an improvement. H.Z. prepared all the figures. All authors contributed to discussion and reviewed the manuscript.

\section{Additional information}

Competing financial interests: The authors declare no competing financial interests.

How to cite this article: Zhang, H., Ma, Z. \& Liu, J.-F. Equilibrium spin current in graphene with Rashba spin-orbit coupling. Sci. Rep. 4, 6464; DOI:10.1038/srep06464 (2014).

This work is licensed under a Creative Commons Attribution-NonCommercialNoDerivs 4.0 International License. The images or other third party material in this article are included in the article's Creative Commons license, unless indicated otherwise in the credit line; if the material is not included under the Creative Commons license, users will need to obtain permission from the license holder in order to reproduce the material. To view a copy of this license, visit http:// creativecommons.org/licenses/by-nc-nd/4.0/ 